\title{
A Real-time Augmented Reality Surgical System for Overlaying Stiffness Information
}

\author{
Nicolas Zevallos*, Rangaprasad Arun Srivatsan, Hadi Salman, Lu Li, \\ Jianing Qian, Saumya Saxena, Mengyun Xu, Kartik Patath and Howie Choset \\ Robotics Institute, Carnegie Mellon University, \\ 5000 Forbes Avenue, Pittsburgh, PA 15213. \\ *Email: nzevallo@andrew.cmu.edu
}

\begin{abstract}
We describe a surgical system that autonomously searches for tumors and dynamically displays a computer graphic model of them super-imposed on the organ (or in our case, phantom). Once localized, the phantom is tracked in real time and augmented with overlaid stiffness information in 3D. We believe that such a system has the potential to quickly reveal the location and shape of tumors, and the visual overlay will reduce the cognitive overload of the surgeon. The contribution of this paper is the integration of disparate technologies to achieve this system. In fact, to the best of our knowledge, our approach is one of the first to incorporate state-of-the-art methods in registration, force sensing and tumor localization into a unified surgical system. First, the preoperative model is registered to the intra-operative scene using a Bingham distribution-based filtering approach. An active level set estimation is then used to find the location and shape of the tumors. We use a recently developed miniature force sensor to perform the palpation. The estimated stiffness map is then dynamically overlaid onto the registered preoperative model of the organ. We demonstrate the efficacy of our system by performing experiments on a phantom prostate model and other silicone organs with embedded stiff inclusions using the da Vinci research kit (dVRK).
\end{abstract}

\section{INTRODUCTION}

Robot-assisted minimally invasive surgeries (RMIS) are becoming increasingly popular as they provide increased dexterity and control to the surgeon while also reducing trauma, blood loss and hospital stays for the patient [23]. These devices are typically teleoperated by the surgeons using visual feedback from stereo-cameras, but without any haptic feedback. This can result in the surgeon relying only on vision to identify tumors by mentally forming the correspondence between intra-operative view and pre-operative images such as CT scans/MRI, which can be cognitively demanding.

Automation of simple but laborious surgical sub-tasks and presenting critical information back to the surgeon in an intuitive manner has the potential to reduce the cognitive overloading and mental fatigue of surgeons [8]. This work leverages the recent advances in force sensing technologies [15], tumor localization strategies [1, 2, 28], online registration techniques [32, 33, 34] and augmented reality [24] to automate the task of tumor localization and dynamically overlay the information on top of intraoperative view of the anatomy.

While there are works in literature that deal with force sensing [25, 37], force-based exploration [29, 31, 39, 40],

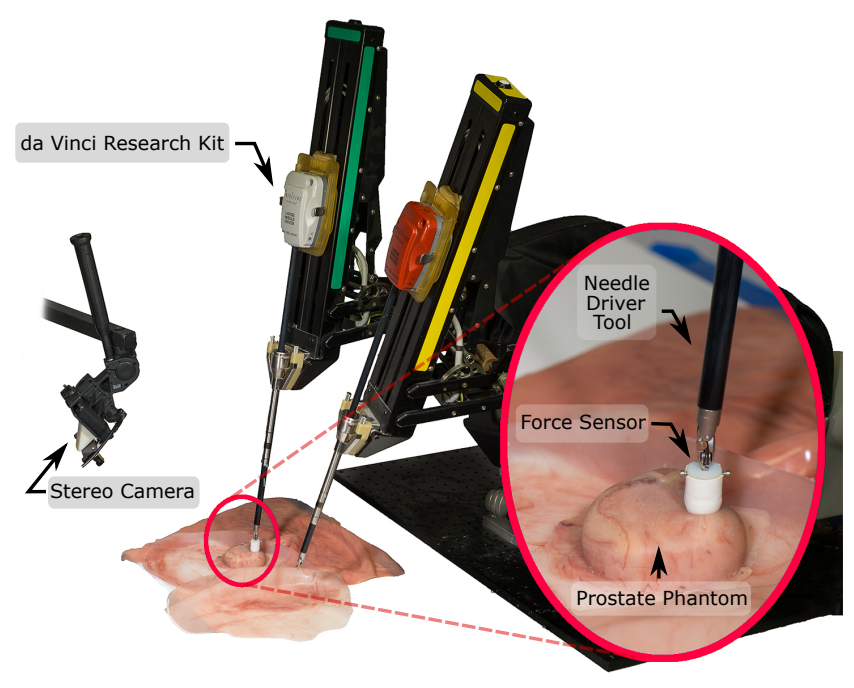

Fig. 1. Experimental setup showing the dVRK robot with a miniature force sensor attached to the end-effector. A stereo camera overlooks the workspace of the robot. A phantom prostate with embedded stiff inclusion is placed in the workspace of the robot.

tumor localization [1, 2, 5, 7, 8, 28] and graphical image overlays [30, 35, 36, 41], there is a gap in literature when it comes to systems that deal with all these issues at the same time. For example, Yamamoto et al. [41] deal with tumor localization and visual overlay, but they assume the registration is known, the organ is flat and place the organ on a force sensing plate, which is not representative of a realistic surgical scenario. On the other hand, Garg et. al. [8] use a palpation probe mounted on a da Vinci research kit (dVRK) tool (This probe was originally developed by McKinley et. al. [18]). However, they do not deal with registering the organ or visual overlay of the estimated stiffness map. The work of Puangmali et. al. [25] (and many others as noted by Tiwana et. al. [37] in their review paper) deal with development of miniature force sensing technologies for minimally invasive surgeries, but they do not discuss strategies to palpate or ways to perform image overlay of the estimated stiffness map. Ayvali et. al. [1, 2] and Salman et. al. [28] develop techniques to smartly palpate a tissue and search for tumors 
with a small number of probings. The work of Chalasani et. al. [5. 7] provides an alternate way of palpation by probing along continuous trajectories. However, these works do not deal with graphically overlaying the estimated stiffness map and also use an ad hoc force sensing set up consisting of a plate mounted on a commercial force sensor. The work of Sanan et. al. [29] and Srivatsan et. al. [31] uses force-based exploration for registration and stiffness estimation, but they do not perform image overlay and spend a lot of time probing the entire organ in a raster-scan pattern. Finally the work of Wang et. al. [39, 40] uses force controlled exploration to perform deformable registration to preoperative model of the organ, but they do not estimate the stiffness of the tissue or perform graphical image overlay. The work in this chapter aims to bridge these shortcomings and present a unified system capable of addressing all the above mentioned issues at the same time.

The system of Naidu et. al. [21] comes closest to our work. They use a custom designed tactile probe (developed by Trejos et. al. [38]) to find tumors and visually overlay the tactile image along with the ultrasound images. The wide tactile array that they use, allows for imaging sections of the organ instead of obtaining discrete measurements, as in our case. This eliminates their need to develop sophisticated tumor search algorithms. However, as acknowledged by Trejos et. al. [38], it is not clear as to how their system would perform when using non-flat organs such as prostates and kidneys; since the tactile array cannot deform and confirm to the shape of the organ. Without performing registration, the image overlay would also be affected on non-flat organs.

The framework presented in this paper is robot agnostic and modular in nature. We demonstrate the efficacy of the system by performing autonomous tumor localization on a phantom prostate model and other custom fabricated flat silicone organs with embedded tumors using the dVRK platform [14] (see Fig. 11. There are two reasons for choosing the dVRK for demonstration - (1) it is a good representation of a surgical robot, (2) there are several research groups across the world that use dVRK and we hope they will all benefit from the work presented in this paper. A miniature force sensor mounted at the tip of the dVRK needle driver tool (developed by Li et. al. [15]) is used to sense the contact forces. An active tumor search strategy developed by Salman et. al. [28] is used to localize the tumor. The estimated stiffness map is overlaid on a registered model of the anatomy and displayed in real-time on a stereo viewer. The overall focus of this paper is to combine the various state-of-the-art methods into a demonstrative surgical system that would enable fast estimation of registration, tumor search and graphical image overlay. A preliminary version of this paper has appeared in [42]. We believe that our contributions would be used in the software framework being developed by our collaborators Chalasani et. al. [6] at Johns Hopkins university and Vanderbilt university to provide online CSA for surgical assistance.

\section{RELATED WORK}

\section{A. Tumor search approaches}

The recent developments in force sensors have also resulted in a number of works that automate mapping of the surface of the anatomy to reveal stiff inclusions. The different palpation strategies commonly used are: discrete probing motion [1. 22, 31, 41], rolling motion [16], cycloidal motion [9] and sinusoidal motion [5, 7]. Some of these works direct the robot along a predefined path that scans the region of interest on the organ [13, 31, 41], while others adaptively change the grid resolution to increase palpation resolution around boundaries of regions with high stiffness gradients [9, 22].

Over the last two years, Bayesian optimization-based methods have gained popularity [1, 2, [5, 7, 8]. These methods model the stiffness map using a Gaussian process regression (GPR) and reduce the exploration time by directing the robot to stiff regions. While the objective of most prior works is to find the high stiffness regions [1, 2, 8], recent work by Salman et al. [28] on active search explicitly encodes finding the location as well as the shape of the tumor as its objective.

\section{B. Surgical registration and image overlay}

There is a rich literature of image overlay for minimally invasive surgeries [30], including some works on usage of augmented reality in human-surgeries [17]. Often the image that is overlaid is a segmented preoperative model, and it manually placed in the intraoperative view [17, 35]. Very few works such as those of Teber et. al. [36] and Haouchine et. al. [11], deal with manual placement followed by automatic registration of the organ models. There are a number of registration techniques that have been developed for surgical applications; the most popular one being iterative closest point (ICP) [3] and its variants [27]. There also exist methods that deal with local deformation caused by tool-tissue interaction [31] and global deformation caused by organ shift, swelling, etc. [20, 40].

Probabilistic methods for registration have recently gained attention as they are better at handling noise in the measurements. Billings et al. [4] use a probabilistic matching criteria for registration, while methods such as [19, 32, 34] ( and the references therein) use Kalman filters to estimate the registration parameters. Recent work by our group reformulates registration as a linear problem in the space of dual quaternions and uses a Bingham filter and a Kalman filter to estimate the rotation and translation respectively [33]. Such an approach has been shown to produce more accurate and fast online updates of the registration parameters.

While the above literature deals with registering preoperative models onto an intraoperative scene, there is very little literature that deals with overlaying stiffness maps on the preoperative models and updating the maps in real-time as new force sensing information is obtained. Real-time updates are very important because they provide the surgeon a better sense of what the robot has found and gives them insight into when to stop the search algorithm which is a subjective decision, as observed by Ayvali et. al. [2].The works of Yamamoto et 
al. [41] and Naidu et al. [21] are exceptions and deal with dynamic overlaying of the stiffness image, but only onto preregistered flat organs. Their approaches do not generalize to the cases of non-flat organs such as kidneys or prostates that we consider in this work.

\section{Force sensing for surgical applications}

The following survey papers report a number of devices that measure contact forces [25, 37]. Some common drawbacks with many existing devices are: difficulty to sterilize, high cost, delicate components and lack of flexibility of form factor. Recently, Li et. al. [15] have developed a miniature force sensor that uses an array of thin-film force-sensitive resistors (FSR) with embedded signal processing circuits. A diagram of the sensor can be seen in Fig. 6(a). This sensor is light weight, inexpensive, robust, and has a flexible form factor.

\section{Problem Setting And Assumptions}

We use an ELP stereo camera (model 1MP2CAM001) overlooking the workspace of a dVRK [14]. A custom fabricated prostate phantom (made using Ecoflex 00-10) as well as two other flat silicone organs, all embedded with plastic pieces to mimic stiff tumors, are used for experimental validation.

Given an a priori geometric model of an organ, the measurements of the tool tip positions and associated contact forces, and stereo-camera images of the intraoperative scene, our goal is to (i) register the camera-frame, robot-frame and modelframe to each other, (ii) estimate the stiffness distribution over the organ's surface, and (iii) overlay the estimated stiffness distribution on the registered model of the organ and display it back to the user.

We make the following assumptions in this work:

- The shape of the organ deforms only locally due to toolinteraction.

- The tool-tip pose can be obtained accurately from the robot kinematics.

- The forces applied by the tool are within the admissible range (less than $10 \mathrm{~N}$ ) in which the organ only undergoes a small deformation (less than $8 \mathrm{~mm}$ ) that allows it to realize its undeformed state when the force is removed.

- The stiff inclusion is located relatively close to the tissue surface, so that it can be detected by palpation.

\section{System Modeling}

Fig. 2 shows the flowchart of the entire system. Modules such as camera calibration, model generation and registration need to be run only once at the beginning of the experiment. On the other hand, the tumor search, probing, and augmented display modules are run in a loop until the user is satisfied with the result and halts the process. While the system is largely autonomous, user input is required in two steps: (i) Cameramodel registration, to select the organ of interest in the view of the camera, (ii) selecting region of interest for stiffness mapping. The modularity of the system allows the user to choose any implementation for registration, force-sensing and tumor localization. The important modules of our system are discussed in detail in the following sections.

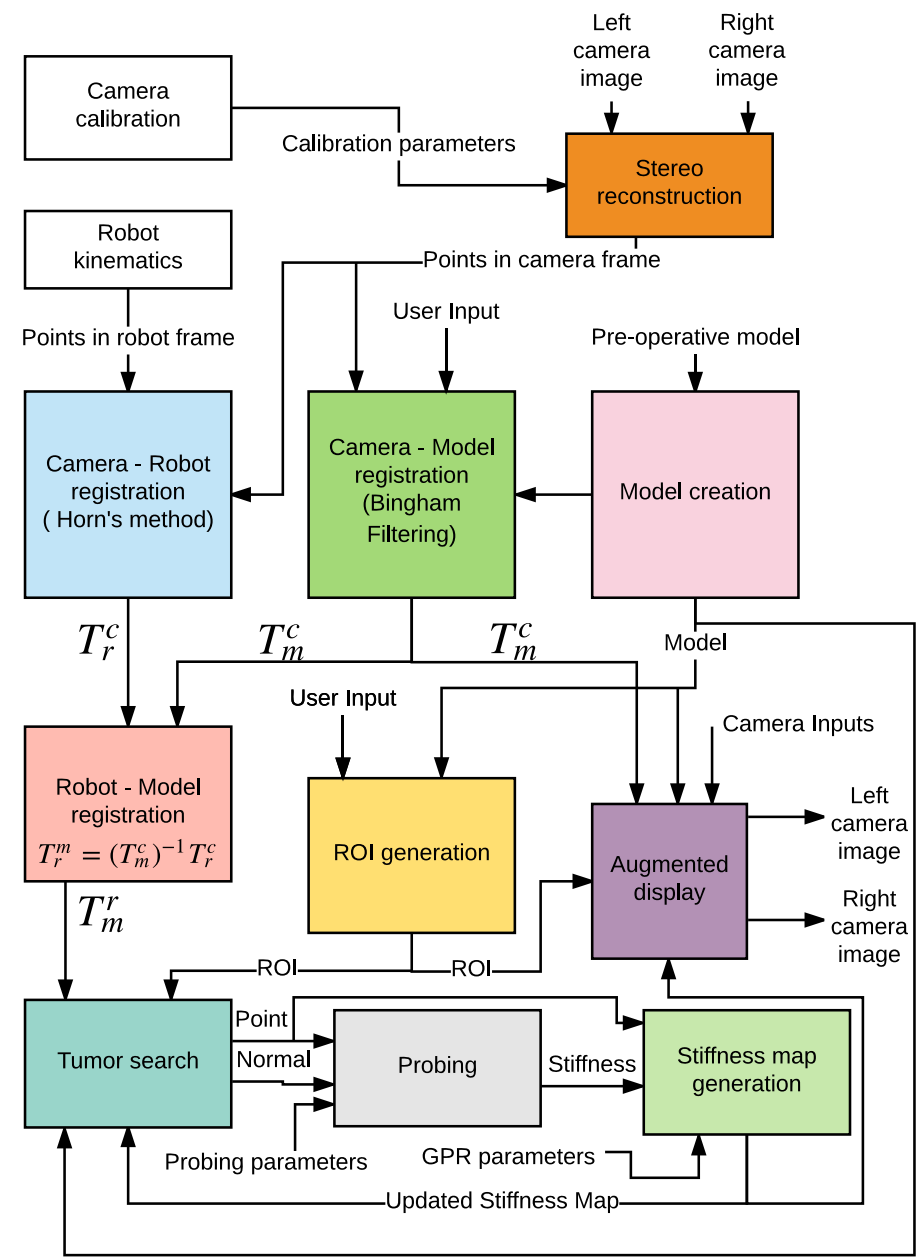

Fig. 2. Flowchart showing all the modular components of our system. Some of the modules such as camera calibration, stereo reconstruction, model creation, and camera-robot-model registrations are performed once before the start of the experiment, while the other modules are constantly run for the duration of the experiment.

\section{A. Registering Camera and Robot Frames}

The cameras are calibrated using standard ROS calibration [26]. The robot is fitted with a colored bead on its end effector that can be easily segmented from the background by hue, saturation, and value. Registration between the cameraframe and the robot-frame is performed by the user through a graphical user interface (GUI) that shows the left and right camera images and has sliders representing color segmentation parameters.

The robot is moved to a fixed set of six points. These points are chosen to cover a substantial amount of the robot's workspace, stay within the field of view in the camera, and not contain symmetries that would make registration difficult. We chose to use only six points after experiments showed that additional points failed to significantly decrease the root mean squared error (RMSE), as shown in Table 1 . For each of the points, we perform a series of actions.

First, we move the robot to the specified location, then we process both the left and right images to find the centroid of 
TABLE I

\begin{tabular}{lcccccc}
\hline Number of points & 5 & 6 & 7 & 8 & 11 & 51 \\
\hline RMSE $(\mathrm{mm})$ & 2.71 & 2.37 & 2.84 & 3.01 & 2.82 & 2.85 \\
\hline
\end{tabular}

the colored bead fitted to the robot. The centroid of the ball in pixels is found as the center of the minimum enclosing circle of the contour with the largest area. We repeat this for as many frames as are received over ROS in one second (in our case 15), and the centroid is then averaged over all frames to reduce the effect of noise in the image. The centroid is drawn onto both images in the GUI, allowing the user to evaluate the accuracy of the centroid estimation. The pixel disparity is calculated as the difference between the $x$ coordinates of the centroid in the left and right images. This disparity is fed into a stereo-camera model that ROS provides, to calculate a 3D point in the camera-frame.

Following this, we obtain six points in both the cameraframe and the robot-frame (using the kinematics of the robot). We use Horn's method [12] to calculate the transformation $\boldsymbol{T}_{m}^{c}$ between the camera and the robot frames. This transformation is saved to a file and the calculated RMSE is displayed to the user. In addition, the robot's current position is transformed by the inverse of the calculated transformation and projected back into the pixel space of both cameras. Circles are drawn at these pixel positions in the left and right images in the GUI so that the user can visually confirm that the registration is successful and accurate.

\section{B. Registering Camera and Preoperative Model Frames}

The transformation between camera-frame and modelframe, $\boldsymbol{T}_{m}^{c}$ is estimated by registering the reconstructed point cloud from stereo images with the preoperative model of the organ. The intraoperative scene as viewed by the stereo cameras is as shown in the top of Fig. 3. A user manually selects the region containing the organ of interest. Following this the user can also further refine the selection using a graph cut-based image segmentation.

A Bingham distribution-based filtering approach is used to automatically register the stereo point cloud to the preoperative model [33, 34]. The mean time taken to register is $2 \mathrm{~s}$ and the RMS error is $1.5 \mathrm{~mm}$. The center row in Fig. 3 shows the registered model of the organ overlaid on the stereo views. Note how the pose of the registered model accurately matches the pose of the organ. In the same figure we also show the model of the tumor in the registered view to highlight how accurately the stiffness map estimates the location of the tumor (see bottom row of Fig. 3)

We also augmented the static grab-cut based segmentation using an automatic traveling mask. Once we are satisfied that the model is roughly registered to the object, we switch from the static graph-cut mask to the traveling mask. Using the same rendering engine used in the GUI, a z-depth buffer is rendered from the camera's view of the model. This depth buffer is scaled from 0 to 255 with 0 representing the pixel
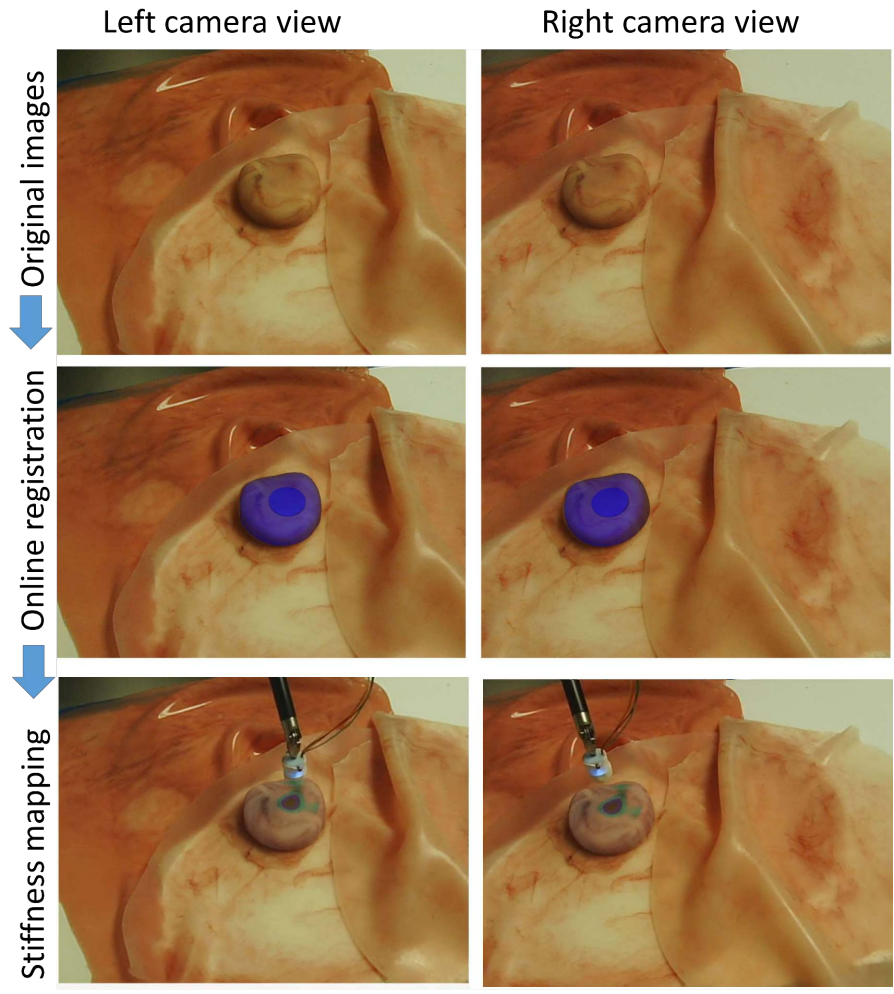

Fig. 3. Top row: Original left and right camera images. Middle row: Camera images with registered prostate model shown in semi-transparent blue. The tumor model is also shown to allow us to compare our stiffness mapping result. Bottom row: The robot probes the organ and records forcedisplacement measurements. The estimated stiffness map is then augmented on the registered model in this figure. Dark blue regions show high stiffness. Note that the stiffness map reveals the location and shape of the tumor.

farthest from the camera and 255 representing the closest. Using this buffer, we create a new mask for our camera image by masking out all pixels with a depth of zero, effectively creating a cutout of our rendered model. Because we render the depth buffer every time the model's estimated transformation is changed, we create an image mask that moves along with our model. Results using this traveling mask can be seen in Fig. 4. Tracking is maintained even after an disturbance moves the silicone prostate out from under the tracked model by approximately $4 \mathrm{~cm}$. Although extreme movements will certainly make our system lose tracking, the system is robust to small movements that may arise due to breathing, heart beat as well as due to interactive forces during palpation. To the best of our knowledge the prior works on probing-based stiffness mapping assume the organ is rigidly clamped with respect to the robot and ignore movement of the organ [7, 21, 31, 41].

\section{Tumor Search and Stiffness Mapping}

The problem of tumor search is often posed as a problem of stiffness mapping, where the stiffness of each point on a certain organ is estimated, and regions with stiffness higher than a certain threshold are considered as regions of interest (tumors, arteries, etc.). The framework that we use for localizing tumors utilizes Gaussian processes (GP) to model the 
Time (a)

RMS: 0.00086435
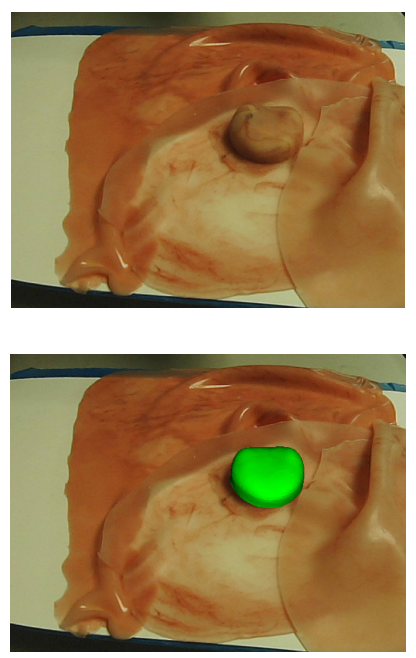

Time (b)

RMS: 0.0021977
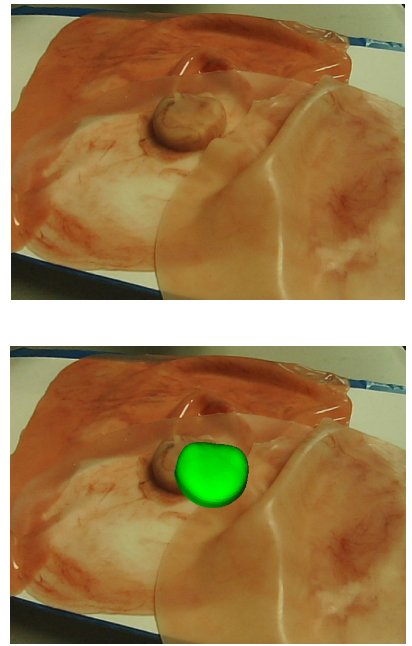

Time (c)

RMS: 0.00087708
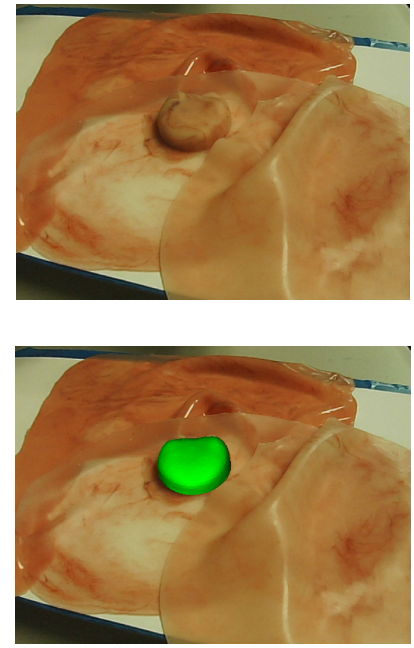

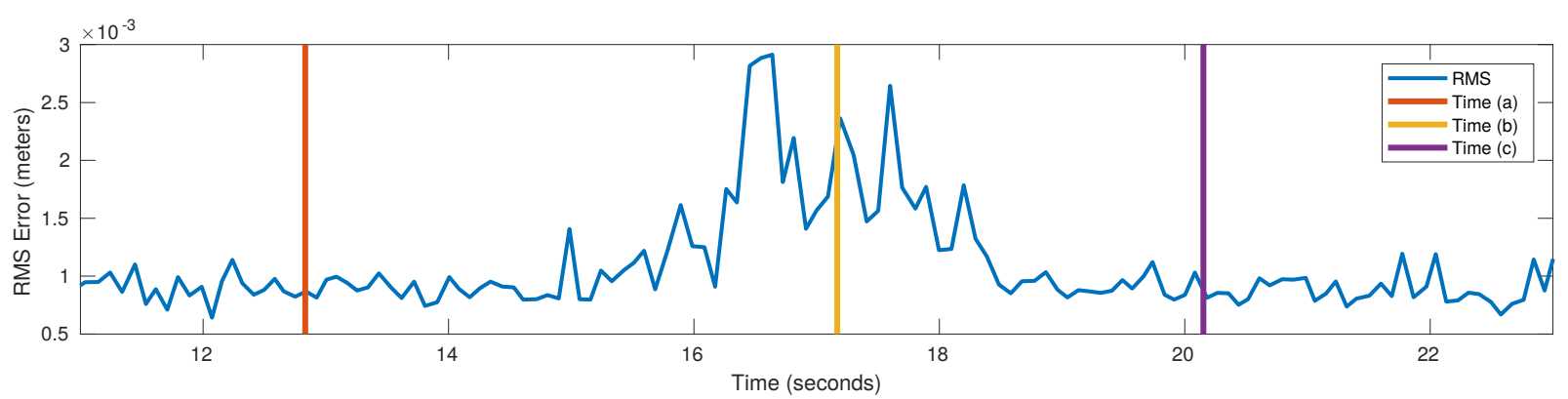

Fig. 4. Registration results on a moving phantom prostate shown at three different times. Note the large disturbance at time (b) caused by applying an external force to the organ and the recovered tracking at time (c).

stiffness distribution combined with a GP-based acquisition function to direct where to sample next for efficient and fast tumor localization. This is described in Fig. 5

By using GP, we assume a smooth change in the stiffness distribution across the organ. Since every point on the organ's surface can be uniquely mapped to a $2 \mathrm{D}$ grid, the domain of search used is $X \subset \mathbb{R}^{2}$. The measured force and position after probing the organ by the robot at $\boldsymbol{x}$ provides the stiffness estimation represented by $y$.

The problem of finding the location and shape of the stiff inclusions can be modeled as an optimization problem. However, an exact functional form for such an optimization is not available in reality. Hence, we maintain a probabilistic belief about the stiffness distribution and define a so called "acquisition function", $\xi_{a c q}$, to determine where to sample next. This acquisition function can be specified in various ways and thus our framework is flexible in terms of the choice of this acquisition function that is being optimized. Prior works have considered various choices for the acquisition functions such as expectation improvement (EI) [1, 2], upper confidence bound (UCB) [8], uncertainty sampling (UNC), active area search (AAS) and active level set estimation (LSE) [28].

While our system is flexible to the choice of acquisition function, in this work we demonstrate tumor localization using LSE. LSE determines the set of points, for which an unknown function (stiffness map in our case) takes value above or below some given threshold level $h$. The mean and covariance of the GP can be used to define a confidence interval,

$$
Q_{t}(\boldsymbol{x})=\left[\mu_{t}(\boldsymbol{x}) \pm \beta^{1 / 2} \sigma_{t}(\boldsymbol{x})\right]
$$

for each point $\boldsymbol{x} \in \bar{X}$. Furthermore, a confidence region $C_{t}$ which results from intersecting successive confidence intervals can be defined as,

$$
C_{t}(\boldsymbol{x})=\bigcap_{i=1}^{t} Q_{i}(\boldsymbol{x}) .
$$

LSE then defines a measure of classification ambiguity $a_{t}(\boldsymbol{x})$ defined as,

$$
a_{t}(\boldsymbol{x})=\min \left\{\max \left(C_{t}(\boldsymbol{x})\right)-h, h-\min \left(C_{t}(\boldsymbol{x})\right)\right\} .
$$

LSE chooses sequentially queries (probes) at $\boldsymbol{x}_{*}$ such that,

$$
\boldsymbol{x}_{*}=\underset{\boldsymbol{x} \in X}{\operatorname{argmax}} a_{t}(\boldsymbol{x}) .
$$




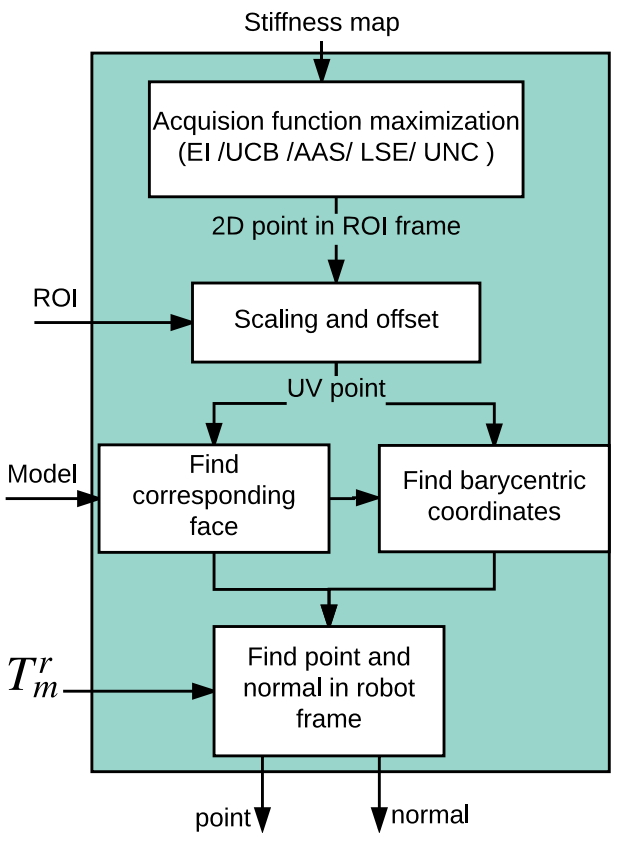

Fig. 5. An expanded flowchart of the "Tumor search" block shown in Fig. 2

For details on how to select the parameter $h$, we refer the reader to the work of Gotovos et al. [10].

\section{Probing and Force Sensing}

We affixed a miniaturized Tri-axial sensor developed in our prior work [15] onto the needle driver tool for the dVRK to provide contact force measurements (see Fig. 1). The force sensor is a Force-Sensitive-Resistor (FSR) based force-to-voltage transducer operating in thru-mode electrodes configuration. The design combines FSR array with a center mounted pre-load mechanical structure to provide a highly responsive measurement of contacting force and direction of the force vector. In this experiment, we electrically bridged the four sensing array elements on the force sensor, to provide improved sensitive force measurement along the normal direction of the sensor, since the dVRK can be accurately oriented to probe along the local surface normal. In addition, we implemented online signal processing software in the sensor embedded controller, for analog signal amplification, filtering, automatic self-calibration, which is crucial step to improve sensor performance when using inexpensive force sensing materials such as $3 \mathrm{M}$ Velostat film from Adafruit

First, the robot is commanded to a safe position $\boldsymbol{p}_{1}$ which is at a known safe height $z_{s a f e}$ as shown in Fig. 6(c). The robot is then commanded to move to position $\boldsymbol{p}_{2}$ which is at an estimated distance $\lambda$ from the desired probing point $\boldsymbol{p}_{0}$, along the normal to the surface at $\boldsymbol{p}_{0}, \boldsymbol{n}$ (see Fig. 6(c)). While maintaining its orientation, the tool is commanded to move to position $\boldsymbol{p}_{3}=\boldsymbol{p}_{2}-\left(\lambda+d_{\max }\right) \boldsymbol{n}$. The force and position data are constantly recorded as the robot moves from $\boldsymbol{p}_{2}$ to $p_{3}$. When the force sensor contacts the tissue surface, if the contact force exceeds a set threshold $F_{\max }$ or if the probe penetrates more than a set depth $d_{\max }$, the robot is no longer moved. This ensures that the probing does not hurt the patient or cause any damage to the robot. Following this we retract the robot to position $\boldsymbol{p}_{2}$ and then $\boldsymbol{p}_{1}$. Note that we do not record force and displacement data during the retraction process.

Next the recorded data is treated as input to the stiffness mapping algorithm similar to [31]. There are two important steps of this algorithm: (i) baseline removal, (ii) stiffness calculation. Ideally, the force sensor reading should be zero when there is no contact between force sensor and the interest area. However, in reality there is always a small residue in the sensor readings even when there is no contact. Thus we find the mean sensor output value when the probe is at $\boldsymbol{p}_{2}$ and then subtract all the subsequent measurements from this baseline force. For stiffness calculation, we use a standard RANSAC algorithm to find the best fit line between the $y$-axis (force sensor data) and $\mathrm{x}$-axis (displacement data). As a result, the calculated regression coefficient indicates the changing rate of the contact force respect to a unit displacement, which can be used as the best approximation of stiffness value (see Fig. 6(b)). Fig. 7 shows the nearly linear variation of force with displacement, justifying the use of slope of the best fit line as an approximation for the stiffness.

\section{E. Dynamic Image Overlay}

The rendering of the overlays is done using the Visualization Toolkit (VTK). Two virtual 3D cameras are created to match the real cameras using the results of camera calibration. The pre-operative model is placed in virtual 3D space according to the camera-to-organ registration, $\boldsymbol{T}_{m}^{c}$, and rendered as a polygonal mesh from the perspective of each camera. These two renders are overlaid onto live video from the left and right camera feeds as their backgrounds. These renderings are displayed in a GUI divided into three tabs. The first tab is for registration, which overlays the pre-operative model as described above and additionally allows the user to mask and segment the point cloud as described in Sec. IV-B. It also provides buttons to start and stop model registration. The second tab allows the user to select a region of interest (ROI) defined in a 2D UV texture map that represents a correspondence between pixels on a $2 \mathrm{D}$ image to $3 \mathrm{D}$ coordinates on the surface of the pre-operative model (see Fig. 7(c)). The third tab overlays the pre-operative model over the camera feeds and allows the user to set the opacity of the overlay using a slider at the bottom of the window.

In addition, the renderings in the third tab add a texture to the rendered model. For this texture, the results of the tumor search are turned into a heat-map image representing relative stiffness in a user-specified region of interest (ROI) (see Fig. 7(c)). This ROI is defined in 2D UV texture coordinates that represent a correspondence between pixels on a $2 \mathrm{D}$ image to $3 \mathrm{D}$ coordinates on the surface of the polygonal mesh. The heat-map image is broadcast over ROS and overlaid onto the pre-operative model's 2D texture image resulting in dark marks in high-stiffness areas while preserving 


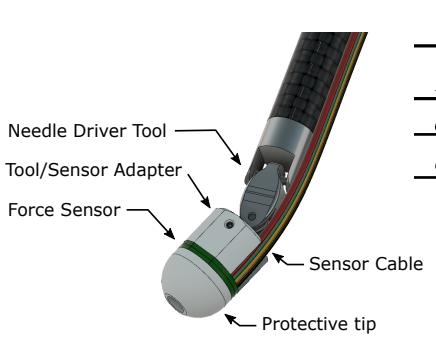

(a)

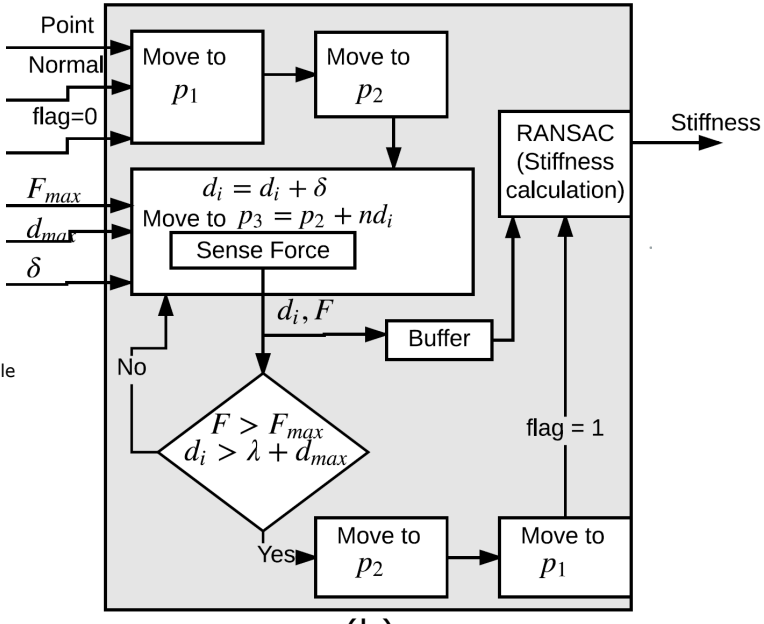

(b)

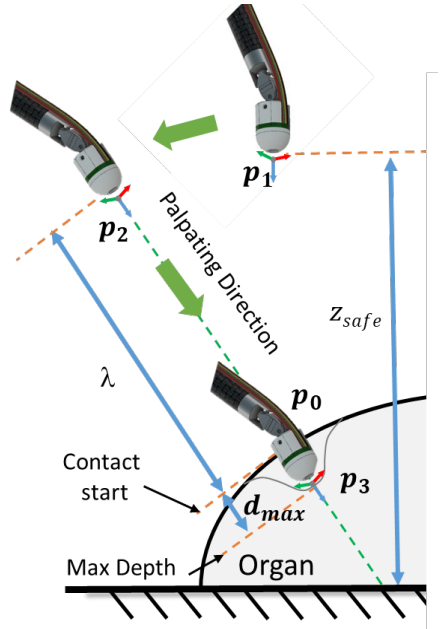

(c)

Fig. 6. (a) The FSR sensor that we use in this paper, was developed by Li et al. [15]. (b) An expanded flowchart of the "Probing" block shown in Fig. 2 (c) The various steps taken to probe a desired point along a desired normal direction as provided by the tumor search module.

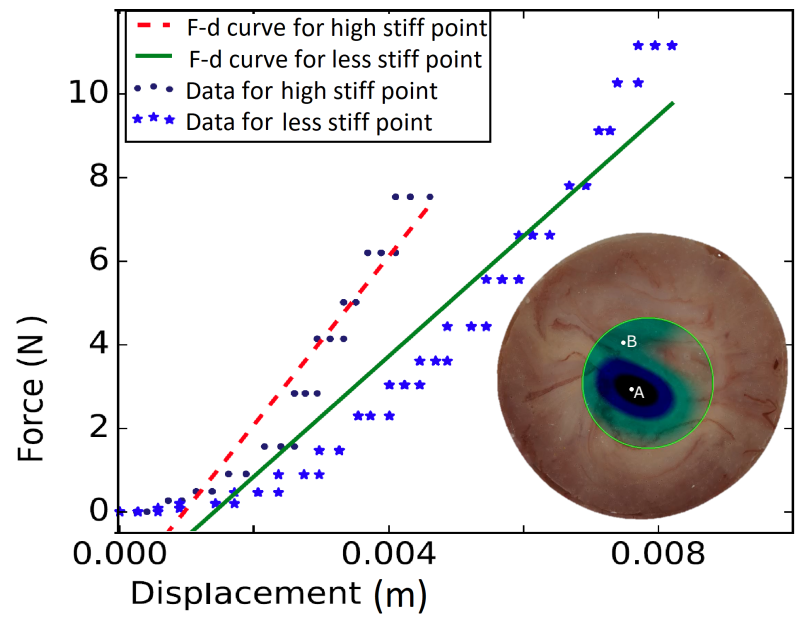

Fig. 7. Force magnitude vs displacement for two sample points A and B on the surface of the organ. RANSAC is used to find the best-fit line and the slope gives us an estimate of the stiffness at the probed location. The circular $2 \mathrm{D}$ space forms a one-to-one mapping with the 3D surface of the organ. The green circle represents the user-defined ROI where stiffness map is estimated. Point $\mathrm{A}$ is located in on a stiff region, while $\mathrm{B}$ is located on a soft region.

texture details found in the pre-operative model's original texture (see Fig. 7(c)). This 2D texture is then applied to the polygonal mesh using the UV map, resulting in a 3D overlay of the stiffness map onto the video feed from each camera. Fig. 8 shows the stiffness maps at various stages of probing, dynamically overlaid on the registered model of the organ. Note that the stiffness map clearly reveals the location and shape of the tumor which is shown in the middle row of Fig. 3 .

\section{EXPERIMENTS AND VALIDATION}

In this section, we validate our system through experimentation on various phantom models. In addition to the experiments performed on the silicone prostate phantom, an experiment
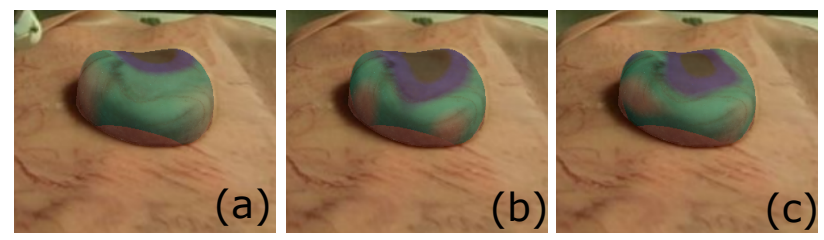

Fig. 8. The figures show the augmented stiffness map at various stages of probing. The high stiffness regions are shown in darker shades of blue, while the low stiffness regions are in lighter shades of blue.(a) Result after 4 probings, (b) result after 18 probings, (c) result after 36 probings.

was performed on a custom fabricated flat silicone organ embedded with plastic inclusions to mimic stiff tumors. We used the dVRK robot with the organ placed on a force sensing plate fitted with a commercial force sensor created by ATI to generate the ground truth stiffness maps.

Upon generating the ground truth stiffness maps using a raster scan pattern with a high density of probed points, the silicone organs were registered and probed using the registration and search methods described in Sec IV] It is worth noting, that unlike Yamamoto et. al. [41], we do not assume the flat organ is pre-registered. We estimate the registration from the stereo-camera and use the estimated registration for the overlay. Also the force sensing plate is only for ground truth stiffness mapping. For the actual experiments, we use the miniature force sensor shown in Fig. 6(a). The resulting stiffness maps, as well as a comparison of how the maps appear when overlaid in our GUI, can be seen in Fig. 9 and 10. These figures show that our system is able to capture the position and size of the tumors with far fewer probed points in a fraction of the time taken to generate the dense stiffness man ${ }^{1}$ Fig. 10 (e) and (f) show the front and back view of the phantom prostate with the overlaid image of the estimated

\footnotetext{
${ }^{1}$ The experiments with our system took a total of 3 minutes to execute as opposed to the raster scan that took upwards of 90 minutes.
} 

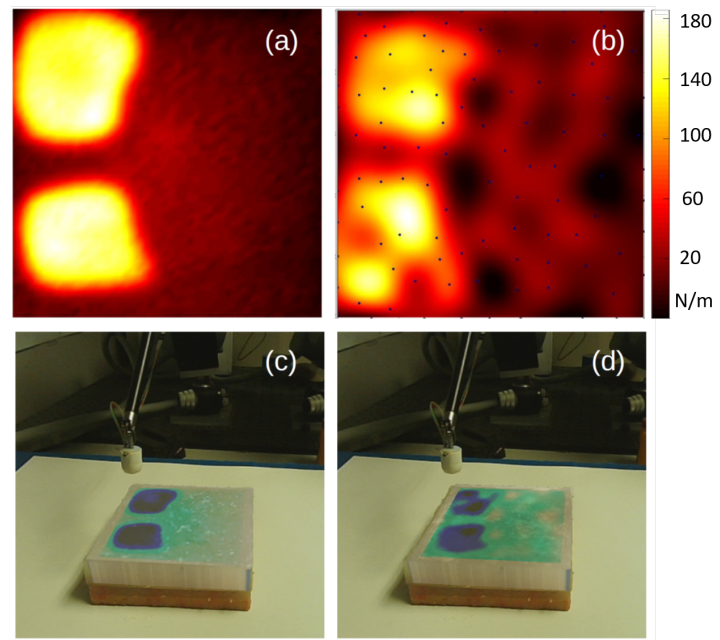

Fig. 9. Experiments on the flat silicone organ. (a) The ground truth stiffness map. (b) The stiffness map as estimated by our system. The probed locations are shown by blue dots. (c) The ground truth stiffness map overlaid on top of the phantom organ. (d) The estimated stiffness map overlaid on top of the phantom organ. The stiffness maps are shown in the space of the UV map. The $x$ and $y$ range for ground truth and estimated stiffness maps are the same.

TABLE II

ACCURACY, RECALL AND PRECISION OF ESTIMATED STIFFNESS MAPS

\begin{tabular}{l|lll} 
& Accuracy $(\%)$ & Recall $(\%)$ & Precision $(\%)$ \\
\hline Prostate & 98.08 & 82.04 & 85.77 \\
Flat organ & 88.50 & 92.11 & 72.21
\end{tabular}

stiffness along with the surface normals at the locations the organ was probed. As is evidenced by this figure, our system is capable of probing and overlaying the image on a non-flat highly curved organ.

Table $\Pi$ II shows the precision, accuracy and recall of the estimated stiffness maps when compared to the ground truth. Precision, accuracy and recall are popular metrics to compare performance of regions-of-interest detection problems and have been used to compare stiffness maps in literature [28]. The results show that the shape and location of tumor as estimated by our approach is accurate and closely matches the ground truth. Although the exact shape of the tumors is not perfectly captured for the case of the flat organ, the resulting map is more than enough to show the user where the tumors are located. The RMS error in the stiffness estimation for the prostate phantom is $18.71 \mathrm{~N} / \mathrm{m}$ and for the flat organ is 40.09 $\mathrm{N} / \mathrm{m}$, which is sufficient for a surgeon to differentiate tumor from tissue as noted by Chalasani et. al. [5, 7].

\section{DisCUSSIONS AND FUTURE WORK}

In this paper, we presented a system that unifies autonomous tumor search with augmented reality to quickly reveal the shape and location of the tumors while visually overlaying that information on the real organ. Our system is capable of probing highly curved organs as well as tracking the movement of the organ that may be caused by the forceful interaction. This has the potential to reduce the cognitive overload of

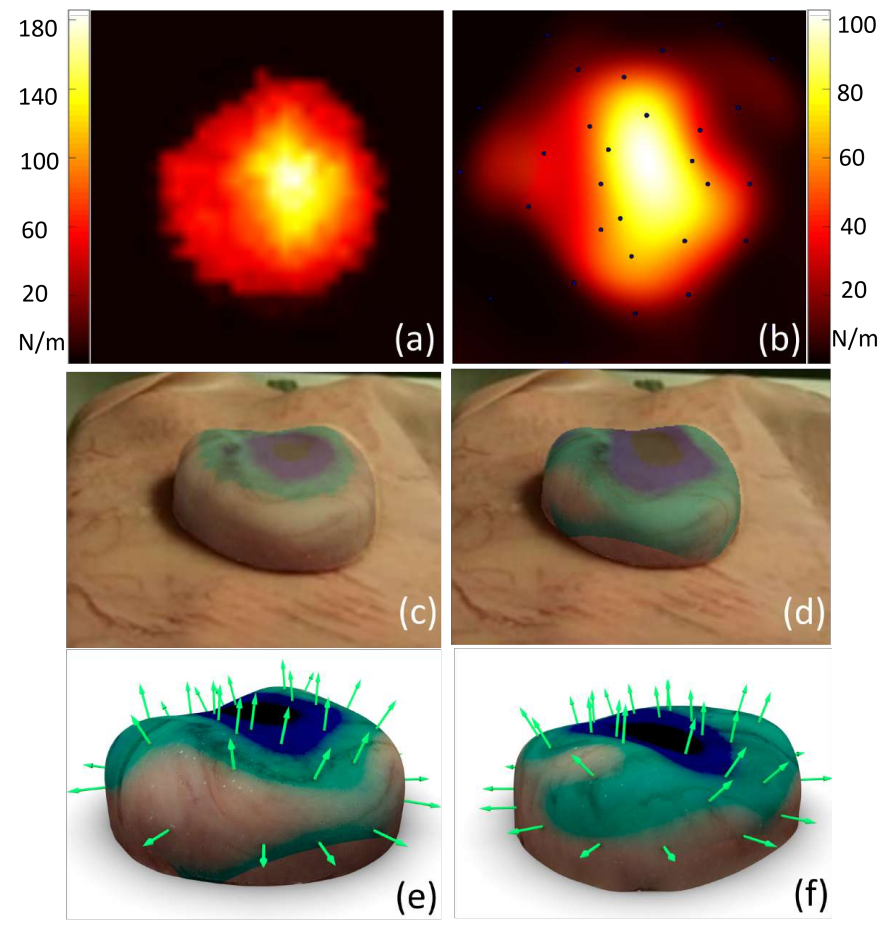

Fig. 10. Experiments on the phantom prostate. (a) The ground truth stiffness map. (b) The stiffness map as estimated by our system. The probed locations are shown by blue dots. (c) The ground truth stiffness map overlaid on top of the phantom organ. The stiffness maps are shown in the space of the UV map. The $x$ and $y$ range for ground truth and estimated stiffness maps are the same. (d) The estimated stiffness map overlaid on top of the phantom organ. (e) and (f) show the front and back view respectively, of the prostate model with an overlay of the estimated stiffness map. The surface normals at the various probed locations are shown by the green arrows.

the surgeons and assist them during the surgery. Our system demonstrates promising results in experimentation on phantom silicone organs.

While we demonstrate the task of stiffness mapping in this work, our system can be used to visually overlay pre-surgical plans, ablation paths, annotate important landmarks, etc. to aid the surgeon during the procedure. In our future work we plan to account for large deformations of the organ and update the model accordingly. We plan to utilize computationally fast approaches to segment the dVRK tools from the images and avoid any obstructions to the overlaid stiffness map. Furthermore, as demonstrated by other researchers in this field, we believe a hybrid force-position controller can result in more accurate probing and hence better stiffness estimation. Finally, we plan to perform experiments on ex-vivo organs and carry user studies to asses the efficacy of the system in a realistic surgical setting.

\section{ACKNOWLEDGMENTS}

This work has been funded through the National Robotics Initiative by NSF grant IIS-1426655 and the Center for Machine Learning and Health. 


\section{REFERENCES}

[1] Elif Ayvali, Rangaprasad Arun Srivatsan, Long Wang, Rajarshi Roy, Nabil Simaan, and Howie Choset. Using Bayesian Optimization to Guide Probing of a Flexible Environment for Simultaneous Registration and Stiffness Mapping. In Proceedings of the International Conference on Robotics and Automation (ICRA), number 10.1109/ICRA.2016.7487225, pages 931-936, 2016.

[2] Elif Ayvali, Alexander Ansari, Long Wang, Nabil Simaan, and Howie Choset. Utility-Guided Palpation for Locating Tissue Abnormalities IEEE Robotics and Automation Letters, 2(2):864-871, 2017.

[3] Paul J Besl and Neil D McKay. Method for registration of 3-D shapes. In Robotics-DL tentative, pages 586-606. International Society for Optics and Photonics, 1992.

[4] Seth D Billings, Emad M Boctor, and Russell H Taylor. Iterative most-likely point registration (IMLP): A robust algorithm for computing optimal shape alignment PloS one, 10(3):e0117688, 2015.

[5] Preetham Chalasani, Long Wang, Rajarshi Roy, Nabil Simaan, Russell H Taylor, and Marin Kobilarov. Concurrent nonparametric estimation of organ geometry and tissue stiffness using continuous adaptive palpation. In ICRA, pages 4164-4171. IEEE, 2016.

[6] Preetham Chalasani, Anton Deguet, Peter Kazanzides, and Russell H Taylor. A Computational Framework for Complementary Situational Awareness (CSA) in Surgical Assistant Robots. In 2018 Second IEEE International Conference on Robotic Computing (IRC), pages 9-16. IEEE, 2018.

[7] Preetham Chalasani, Long Wang, Rashid Yasin, Nabil Simaan, and Russell H Taylor. Preliminary Evaluation of an Online Estimation Method for Organ Geometry and Tissue Stiffness IEEE Robotics and Automation Letters, 3(3):1816-1823, 2018.

[8] Animesh Garg, Siddarth Sen, Rishi Kapadia, Yiming Jen, Stephen McKinley, Lauren Miller, and Ken Goldberg. Tumor localization using automated palpation with gaussian process adaptive sampling. In proceedings of International Conference on Automation Science and Engineering (CASE), pages 194-200. IEEE, 2016.

[9] Roger E Goldman, Andrea Bajo, and Nabil Simaan. Algorithms for autonomous exploration and estimation in compliant environments Robotica, 31(1):71-87, 2013.

[10] Alkis Gotovos, Nathalie Casati, Gregory Hitz, and Andreas Krause. Active learning for level set estimation In IJCAI, pages 1344-1350, 2013.

[11] Nazim Haouchine, Jeremie Dequidt, Igor Peterlik, Erwan Kerrien, Marie-Odile Berger, and Stephane Cotin. Towards an accurate tracking of liver tumors for augmented reality in robotic assisted surgery. In ICRA, pages 41214126, 2014.

[12] Berthold KP Horn. Closed-form solution of absolute orientation using unit quaternions. JOSA A, 4(4):629642, 1987.
[13] Robert D Howe, William J Peine, DA Kantarinis, and Jae S Son. Remote palpation technology, IEEE Engineering in Medicine and Biology Magazine, 14(3):318323, 1995.

[14] Peter Kazanzides, Zihan Chen, Anton Deguet, Gregory S Fischer, Russell H Taylor, and Simon P DiMaio. An open-source research kit for the da Vinci ${ }^{\circledR}$ Surgical System. In ICRA, pages 6434-6439. IEEE, 2014.

[15] Lu Li, Bocheng Yu, Chen Yang, Prasad Vagdargi, Rangaprasad Arun Srivatsan, and Howie Choset. Development of an Inexpensive Tri-axial Force Sensor for Minimally Invasive Surgery. In In proceedings of the International Conference on Intelligent Robots and Systems (IROS). IEEE, 2017.

[16] Hongbin Liu, David P Noonan, Benjamin J Challacombe, Prokar Dasgupta, Lakmal D Seneviratne, and Kaspar Althoefer. Rolling mechanical imaging for tissue abnormality localization during minimally invasive surgery IEEE Transactions on Biomedical Engineering, 57:404414, 2010.

[17] Jacques Marescaux, Francesco Rubino, Mara Arenas, Didier Mutter, and Luc Soler. Augmented-reality-assisted laparoscopic adrenalectomy Jama, 292(18):2211-2215, 2004.

[18] Stephen McKinley, Animesh Garg, Siddarth Sen, Rishi Kapadia, Adithyavairavan Murali, Kirk Nichols, Susan Lim, Sachin Patil, Pieter Abbeel, Allison M Okamura, et al. A single-use haptic palpation probe for locating subcutaneous blood vessels in robot-assisted minimally invasive surgery. In proceedings of International Conference on Automation Science and Engineering (CASE), pages 1151-1158. IEEE, 2015.

[19] Mehdi Hedjazi Moghari and Purang Abolmaesumi. Point-based rigid-body registration using an unscented Kalman filter. IEEE Transactions on Medical Imaging, 26(12):1708-1728, 2007.

[20] A. Myronenko and Xubo Song. Point Set Registration: Coherent Point Drift. IEEE Transactions on Pattern Analysis and Machine Intelligence, 32(12):2262-2275, Dec 2010. ISSN 0162-8828. doi: 10.1109/TPAMI.2010. 46.

[21] Anish S Naidu, Michael D Naish, and Rajni V Patel. A Breakthrough in Tumor Localization IEEE Robotics \& Automation Magazine, 1070(9932/17), 2017.

[22] Kirk A Nichols and Allison M Okamura. Methods to segment hard inclusions in soft tissue during autonomous robotic palpation IEEE Transactions on Robotics, 31(2): 344-354, 2015.

[23] Jaydeep Palep. Robotic assisted minimally invasive surgery. Journal of Minimal Access Surgery, 5(1):1-7, Jan 2009.

[24] Kartik Patath, Rangaprasad Arun Srivatsan, Nicolas Zevallos, and Howie Choset. Dynamic Texture Mapping of 3D models for Stiffness Map Visualization In Workshop on Medical Imaging, IEEE/RSJ International Conference on Intelligent Robots and Systems (IROS), 2017. 
[25] Pinyo Puangmali, Kaspar Althoefer, Lakmal D Seneviratne, Declan Murphy, and Prokar Dasgupta. State-ofthe-art in force and tactile sensing for minimally invasive surgery, IEEE Sensors Journal, 8(4):371-381, 2008.

[26] Morgan Quigley, Ken Conley, Brian Gerkey, Josh Faust, Tully Foote, Jeremy Leibs, Rob Wheeler, and Andrew Y Ng. ROS: an open-source Robot Operating System. In ICRA workshop on open source software, volume 3, page 5. Kobe, Japan, 2009.

[27] Szymon Rusinkiewicz and Marc Levoy. Efficient variants of the ICP algorithm. In Proceedings of 3rd International Conference on 3-D Digital Imaging and Modeling, pages 145-152. IEEE, 2001.

[28] Hadi Salman, Elif Ayvali, Rangaprasad Arun Srivatsan, Yifei Ma, Nicolas Zevallos, Rashid Yasin, Long Wang, Nabil Simaan, and Howie Choset. Trajectory-Optimized Sensing for Active Search of Tissue Abnormalities in Robotic Surgery. In proceedings of International Conference on Robotics and Automation (ICRA). IEEE, 2018.

[29] Siddharth Sanan, Stephen Tully, Andrea Bajo, Nabil Simaan, and Howie Choset. Simultaneous Compliance and Registration Estimation for Robotic Surgery. In Proceedings of the Robotics: Science and Systems Conference, 2014.

[30] Jeffrey $\mathrm{H}$ Shuhaiber. Augmented reality in surgery. Archives of surgery, 139(2):170-174, 2004.

[31] Rangaprasad Arun Srivatsan, Elif Ayvali, Long Wang, Rajarshi Roy, Nabil Simaan, and Howie Choset. Complementary Model Update: A Method for Simultaneous Registration and Stiffness Mapping in Flexible Environments In Proceedings of the International Conference on Robotics and Automation (ICRA), pages 924-930, 2016.

[32] Rangaprasad Arun Srivatsan, Gillian T Rosen, Feroze D Naina, and Howie Choset. Estimating SE(3) elements using a dual quaternion based linear Kalman filter In Robotics : Science and Systems, 2016.

[33] Rangaprasad Arun Srivatsan, Mengyun Xu, Nicolas Zevallos, and Howie Choset. Bingham Distribution-Based Linear Filter for Online Pose Estimation. In Robotics : Science and Systems (RSS), 2017.

[34] Rangaprasad Arun Srivatsan, Mengyun Xu, Nicolas Zevallos, and Howie Choset. Probabilistic pose estimation using a bingham distribution- based linear filter. The International Journal of Robotics Research (IJRR), 2018.

[35] Li-Ming Su, Balazs P Vagvolgyi, Rahul Agarwal, Carol E Reiley, Russell H Taylor, and Gregory D Hager. Augmented reality during robot-assisted laparoscopic partial nephrectomy: toward real-time 3D-CT to stereoscopic video registration. Urology, 73(4):896-900, 2009.

[36] Dogu Teber, Selcuk Guven, Tobias Simpfendörfer, Mathias Baumhauer, Esref Oguz Güven, Faruk Yencilek, Ali Serdar Gözen, and Jens Rassweiler. Augmented reality: a new tool to improve surgical accuracy during laparoscopic partial nephrectomy? Preliminary in vitro and in vivo results. European urology, 56(2):332-338, 2009.
[37] Mohsin I Tiwana, Stephen J Redmond, and Nigel H Lovell. A review of tactile sensing technologies with applications in biomedical engineering. Sensors and Actuators A: physical, 179:17-31, 2012.

[38] Ana Luisa Trejos, Jagadeesan Jayender, MT Perri, Michael D Naish, Rajnikant V Patel, and RA Malthaner. Robot-assisted tactile sensing for minimally invasive tumor localization. The International Journal of Robotics Research (IJRR), 28(9):1118-1133, 2009.

[39] Long Wang, Zihan Chen, Preetham Chalasani, Jason Pile, Peter Kazanzides, Russell H Taylor, and Nabil Simaan. Updating virtual fixtures from exploration data in forcecontrolled model-based telemanipulation. In ASME 2016 International Design Engineering Technical Conferences and Computers and Information in Engineering Conference, pages V05AT07A031-V05AT07A031. American Society of Mechanical Engineers, 2016.

[40] Long Wang, Zihan Chen, Preetham Chalasani, Rashid M Yasin, Peter Kazanzides, Russell H Taylor, and Nabil Simaan. Force-Controlled Exploration for Updating Virtual Fixture Geometry in Model-Mediated Telemanipulation Journal of Mechanisms and Robotics, 9(2): 021010, 2017.

[41] Tomonori Yamamoto, Balazs Vagvolgyi, Kamini Balaji, Louis L Whitcomb, and Allison M Okamura. Tissue property estimation and graphical display for teleoperated robot-assisted surgery. In ICRA, pages 4239-4245, 2009.

[42] Nicolas Zevallos, Rangaprasad Arun Srivatsan, Hadi Salman, Lu Li, Jianing Qian, Saumya Saxena, Mengyun $\mathrm{Xu}$, Kartik Patath, and Howie Choset. A surgical system for automatic registration, stiffness mapping and dynamic image overlay In International Symposium on Medical Robotics (ISMR), pages 1-6. IEEE, 2018. 